\title{
Emerging HIV-associated concerns: osteopenia and osteoporosis. Easy prevention and management guidelines
}

Roberto Manfredi

From $16^{\text {th }}$ International Symposium on HIV and Emerging Infectious Diseases

Marseille, France. 24-26 March 2010

\section{Background}

Osteopenia and osteoporosis $(\mathrm{O})$ are emerging complications of HIV infection, especially when treated with combined antiretroviral therapy (cART). The pathogenesis is multifactorial, potentially involving all classes of anti-HIV drugs, although protease inhibitor (PI) use, overall HIV and CART duration, and the male sex, seem related to a significantly greater risk.

\section{Methods}

In a preliminary DEXA screening assessing lumbar spine and femoral head of around 100 out of around 1,000 single-centre patients (p), the frequency of osteopeniaosteoporosis (based on lumbar T-score) was assessed around $48 \%$. An increased risk was found in p treated with protease inhibitors versus $\mathrm{p}$ receiving non-nucleoside reverse transcriptase inhibitor, or triple nucleos $(\mathrm{t})$ ide reverse transcriptase inhibitors.

\section{Results}

Prospective studies of extensive p samples are needed, to elucidate the epidemiology, pathogenesis, clinical issues, and evolution of HIV-associated bone metabolism abnormalities. When planning strategies for their early diagnosis, prevention, and management, also costeffectiveness issues should be taken into consideration, since no pharmacoeconomic data still exist in this setting. Although severe consequences (pathological fractures, prosthetic implants), are expected to be proportionally infrequent events, their consequences in terms of length-intensity of hospitalization, related costs, and especially consequences on the patient's quality of life, are expected to play a remarkable role. Anyway, the most reliable diagnostic procedure of O (i.e. DEXA scan), has affordable costs (around Eur 43.40 for a scan which also offers a body composition assessment), as well as the first-line drugs for osteopenia, e.g. supplementation with calcium (Eur 6/month), and vitamin D (Eur $7 /$ month). These costs cannot be compared with the standards costs of an asymptomatic cART-treated $p$ (Eur 471 to $874 /$ month), and the immunologic, virologic, laboratory, and clinical controls made at least quarterly in the same $\mathrm{p}$.

\section{Discussion}

Like post-menopausal O, also HIV disease should be investigated from multiple cost-effectiveness points of view, to establish which $\mathrm{p}$ are the early candidates for a DEXA screening, when this examination is more useful during HIV disease course-therapy, when the DEXA scan should be repeated, and when-how to intervene pharmacologically, in order to prevent serious and potentially invalidating O-related complications.

Published: 11 May 2010

Cite this article as: Manfredi: Emerging HIV-associated concerns: osteopenia and osteoporosis. Easy prevention and management guidelines. Retrovirology 2010 7(Suppl 1):P68.

Correspondence: Roberto.manfredi@unibo.it

Infectious Diseases, University of Bologna, S. Orsola Hospital, Bologna, Italy 